\title{
¿Cómo aumentar el consumo de leguminosas en la población Chilena?
}

\section{How can legume consumption be increased in the Chilean population?}

\section{RESUMEN}

En las últimas décadas la obesidad se ha transformado en uno de los mayores problemas de salud pública a nivel mundial. En Chile más del $50 \%$ de los niños de pre kínder y $1^{\circ}$ año básico tienen exceso de peso. Esto ha motivado acciones para fomentar la alimentación saludable, como la Ley 20.606, las Guías Alimentarias Chilenas del año 2013 y la incorporación de alimentos saludables en el Programa de Alimentación Escolar. A pesar de lo anterior, se observó un aumento del consumo aparente de alimentos procesados de alta densidad energética, grasas saturadas, sodio y de azúcares agregados, perdiendo importancia los alimentos naturales (verduras, frutas y legumbres). Las legumbres han presentado un consumo decreciente desde 1987, llegando a consumir menos de kilo/hogar al mes. Por otro lado, existe una clara aceptación del consumo de legumbres en los niños, a pesar de desconocer las recomendaciones de consumo, y un rechazo a su preparación en el hogar por las madres, indicando el poco apoyo del hogar a la adquisición de este saludable hábito en los niños. A pesar de las estrategias establecidas para aumentar el consumo de legumbres, estas han sido escasas y con baja cobertura. Se ha demostrado que la escuela es importante en el desarrollo de estilos de vida saludables, al igual que un ambiente familiar que los fomente.

Palabras Clave: Obesidad; Educación Alimentaria Nutricional; Hábitos alimentarios; Legumbres; Marketing Social.

\section{ABSTRACT}

In recent decades, obesity has become one of the biggest public health problems worldwide. In Chile, more than $50 \%$ of pre-school and 1st-grade students are overweight. This has led to a series of actions to promote healthy eating in the Chilean population, such as the 20,606 law, the 2013 Chilean Dietary Guidelines and the incorporation of healthy foods into the school food program. Despite these efforts there has been an increase in the consumption of high energy density processed foods, saturated fats, sodium and added sugars, and a decrease in the consumption of natural foods (vegetables, fruits and legumes). Legume
Sonia Olivares, Natalia Rossi, Nelly Bustos.

Instituto de Nutrición y Tecnología de los Alimentos, Universidad de Chile.

Dirigir correspondencia a: Nelly Bustos, Unidad de Nutrición Pública, Instituto de Nutrición y Tecnología de los Alimentos, Universidad de Chile, Av. El Líbano 5524, Macul, Santiago, Chile. E-mail nbustos@inta.uchile.cl

Este trabajo fue recibido el 15 de junio de 2017. Aceptado para ser publicado: 15 de diciembre de 2017

consumption has been decreasing since 1987, with current consumption averaging less than 1 kilogram per home per month. On the other hand, there is a clear acceptance of legume consumption by children, despite not knowing recommended consumption. At the same time, mothers demonstrate a rejection to legume preparation in the home; indicating how little support there is in the home for children to acquire this healthy eating habit. Strategies to increase the consumption of legumes have been few and have had low coverage. It has been shown that the school environment is important in developing healthy lifestyles in children, as 
is a family environment that promotes healthy lifestyles.

Key words: Obesity; Food Nutrition Education; eating habits; Legumes; Social Marketing.

\section{INTRODUCCIÓN}

Los grandes cambios socioeconómicos, demográficos y ambientales observados en el mundo en las últimas décadas, se han traducido en una transición epidemiológica y nutricional que en América del Sur ha significado un marcado cambio en la estructura de la dieta y la distribución de la composición corporal'. En Chile, la transición se ha caracterizado por una rápida reducción de la fecundidad y de las enfermedades materno infantiles, así como por el envejecimiento de la población y el aumento progresivo de las enfermedades crónicas no trasmisibles (ENT), especialmente enfermedades cardiovasculares, diabetes y cáncer, en el marco de una elevada prevalencia de sobrepeso y obesidad.

La obesidad se ha transformado en uno de los mayores problemas de salud pública a nivel mundial, con una alta proporción de países que presentan más de un 30\% de su población con exceso de peso ${ }^{3}$. El informe 2015 de la Comisión de la Organización Mundial de la Salud (OMS) para acabar con la Obesidad Infantil, señala que en todo el mundo el número de lactantes y niños menores de 5 años que padecen sobrepeso u obesidad aumentó de 32 millones en 1990 a 42 millones en 2013, cifra que podría alcanzar los 70 millones el 2025 si se mantienen las tendencias actuales ${ }^{4}$.

En las últimas 3 décadas se ha observado un incremento progresivo del sobrepeso y obesidad infantil a nivel mundial, tanto en los países desarrollados como en los en vías de desarrollo5 ${ }^{5}$. En Chile, el año 2016 la Junta Nacional de Auxilio Escolar y Becas (JUNAEB), en su Mapa Nutricional de los niños que se incorporan a la educación preescolar o a primer año básico, detectó que el 50,3\% de los niños asistentes a pre-kínder y el 51,1\% de los asistentes a kínder en la Junta Nacional de Jardines Infantiles (JUNJI), respectivamente, presentaba sobrepeso u obesidad, cifra que alcanzó al 51,2\% en los que ingresaron a primer año básico en las escuelas públicas. En todas las categorías, los niños con sobrepeso y obesidad superan a la cantidad de alumnos con peso normal que están en esta etapa escolar ${ }^{6}$.

Como resultado de la urbanización, la exposición a entornos obesogénicos va en aumento, tanto en los países de ingresos altos como en los de ingresos bajos y medianos y en todos los grupos socioeconómicos ${ }^{4}$. Los cambios en el tipo de alimentos y su disponibilidad ${ }^{7,8}$, sumado a la publicidad de alimentos ${ }^{9}$, el aumento en el tamaño de las porciones y el sedentarismo, entre otros, constituyen las principales causas del desequilibrio energético conducente a esta situación ${ }^{4}$.

Aunque en el país, el 27 de junio de 2016 se comenzó a implementar la Ley 20.606, que controla la venta y publicidad de alimentos procesados con alto contenido de calorías y nutrientes críticos (grasas saturadas, azúcares y sodio), y además limita su venta y consumo en los jardines infantiles y escuelas ${ }^{10}$, es posible seguir consiguiendo los alimentos procesados a un bajo costo en el comercio establecido e informal ${ }^{11,12}$.

\section{Patrones de consumo y recomendaciones de legumbres en la población chilena}

Los hábitos y prácticas alimentarias de una población, también denominados patrones alimentarios, pueden verse modificados por el ambiente, por la familia o por diversas variables que interactúan entre sí, entre las que destacan el aumento de la capacidad de compra, el acceso a los alimentos, la globalización de la economía y la alimentación, las estrategias de marketing, entre otros factores que han contribuido a los cambios en el patrón alimentario en todos los quintiles de ingreso, aumentando el consumo aparente de alimentos procesados de alta densidad energética, grasas saturadas, sodio y de azúcares agregados, perdiendo importancia los alimentos naturales, fuentes de fibra dietaria y fitoquímicos como las verduras, frutas y legumbres ${ }^{13}$.

Estudios realizados entre los años 1988 y 2012, observaron un aumento en el gasto absoluto en la mayoría de los grupos de alimentos, lo que se observó en los huevos, aceites, grasas y legumbres, siendo estas últimas las que tuvieron una tendencia decreciente observada desde 1987, donde los hogares no alcanzan a gastar un kilo de legumbres al mes, a diferencia de las hortalizas y frutas donde se observó un aumento en todos los hogares ${ }^{14,15}$.

Considerando lo anterior, las estrategias utilizadas para aumentar el consumo de legumbres en la población chilena han sido escasas y con baja cobertura. Un ejemplo lo constituyen las Guías Alimentarias para la Población Chilena, publicadas el año 2013, respaldadas con la Resolución Exenta № 260, del Ministerio de Salud, que aprueba la Norma General Técnica № 148 sobre Guías alimentarias para la población ${ }^{16}$. Dichas Guías incluyen el mensaje "Consume legumbres al menos dos veces por semana, sin mezclarlas con cecinas".

Por otra parte, estudios realizados en escolares han demostrado un bajo conocimiento por parte de los niños en lo que respecta a la identificación de las porciones de consumo recomendadas por las Guías Alimentarias Chilenas del 2013, siendo las recomendaciones menos conocidas las de frutas $(54,4 \%)$, verduras $(66,5 \%)$ y legumbres $(64,7 \%)^{17}$.

Frente al consumo de alimentos, los resultados de un estudio mostraron que los niños identificaban en un mayor porcentaje las recomendaciones de las GABA, sin embargo, al momento de practicarlas, más del 50\% de ellos tenían un bajo consumo de alimentos saludables, como el agua que no superaba el $13 \%$, el pescado que alcanzaba un $10 \%$, las frutas un $38,2 \%$ y las legumbres un $48 \%{ }^{17}$. Estos resultados coinciden con lo encontrado en un estudio anterior, donde se observó un bajo consumo de alimentos saludables y un elevado consumo de bebidas azucaradas y otros alimentos con altos contenidos de grasa, azúcar y sal, en niñas de bajo NSE, que se diferenciaba del consumo en niñas de alto NSE ${ }^{8}$.

Diversos estudios han demostrado que los patrones de 
alimentación de los niños están fuertemente influenciadas principalmente por factores familiares y sociales ${ }^{18,19}$, y señalan que las prácticas de alimentación materna desempeñan un papel importante en el desarrollo del aumento de peso y comportamientos alimentarios obesogénicos en niños de 2 a 4 años $^{20}$, mientras que en niños de 4 a 12 años, una revisión sistemática señaló que los padres pueden influenciar a los niños en sus tendencias alimentarias ${ }^{21}$. Sin embargo, estas asociaciones proceden de estudios transversales, por lo que es difícil sacar conclusiones al respecto, para determinar la naturaleza recíproca de la crianza y el efecto de las prácticas alimentarias en el IMC en los niños de este rango de edad ${ }^{22}$.

Frente a esta realidad, la OMS y la FAO, ya el año 2003, en su publicación sobre Dieta, Nutrición y Prevención de las Enfermedades Crónicas, señalaron que para mejorar los hábitos alimentarios de los niños se requieren cambios en el ambiente familiar y escolar, eliminando las bebidas azucaradas, mejorando la variedad de alimentos saludables y disminuyendo la oferta de alimentos de alta densidad energética ${ }^{23}$.

\section{Estrategias para mejorar los hábitos alimentarios de la población chilena}

El actual perfil epidemiológico de la población chilena, ha convertido a las Guías Alimentarias en un instrumento de comunicación y educación de gran importancia para orientar a la población en la selección de alimentos saludables, con el fin de ayudarla a identificar y seguir las recomendaciones alimentarias para una buena nutrición y salud. Las GABA son consideradas un aporte esencial al logro de las metas establecidas por la OMS en la Estrategia Mundial sobre Régimen Alimentario, Actividad Física y Salud 2004, que intentan contribuir a que las personas logren un equilibrio energético y un peso normal; limiten la ingesta energética procedente de grasas, sustituyan las grasas saturadas por insaturadas; traten de eliminar los ácidos grasos trans; aumenten el consumo de frutas y hortalizas, legumbres, cereales integrales y frutos secos; limiten la ingesta de azúcares libres y la ingesta de sal ${ }^{3}$.

Las recomendaciones de legumbres en las Guías Alimentarias para la población chilena han estado presentes desde el año 1997, con el mensaje "Aumente el consumo de frutas, verduras y legumbres" 24,25 ; el año 2005 se modificó a "Come porotos, garbanzos, lentejas o arvejas al menos dos veces por semana, en reemplazo de la carne ${ }^{\prime 26}$, y el año 2013 "Consume legumbres al menos dos veces por semana, sin mezclarlas con cecinas" ${ }^{\prime \prime 16}$.

Las Guías Alimentarias para la Población Chilena vigentes desde el año 2013 son las siguientes:

1. Para tener un peso saludable, come sano y realiza actividad física diariamente.

2. Pasa menos tiempo frente al computador o la tele y camina a paso rápido, mínimo 30 minutos al día.

3. Come alimentos con poca sal y saca el salero de la mesa.
4. Si quieres tener un peso saludable, evita el azúcar, dulces, bebidas y jugos azucarados.

5. Cuida tu corazón evitando las frituras y alimentos con grasas como cecinas y mayonesa.

6. Come 5 veces verduras y frutas frescas de distintos colores, cada día.

7. Para fortalecer tus huesos, consume 3 veces en el día lácteos bajos en grasa y azúcar.

8. Para mantener sano tu corazón, come pescado al horno o a la plancha, 2 veces por semana.

9. Consume legumbres al menos dos veces por semana, sin mezclarlas con cecinas.

10. Para mantenerte hidratado, toma 6 a 8 vasos de agua al día.

11. Lee y compara las etiquetas de los alimentos y prefiere los que tengan menos grasas, azúcar y sal (sodio).

\section{Programa de Alimentación Escolar (PAE), a cargo de la Junta Nacional de Auxilio Escolar y Becas (JUNAEB)}

Este programa beneficia diariamente a 1 millón 800 mil niñas, niños y jóvenes del país, a través de la entrega de raciones alimenticias que incluyen desayunos, almuerzos, cenas y colaciones a los alumnos y alumnas en condición de vulnerabilidad de Establecimientos Educacionales Municipales y Particulares Subvencionados adscritos al Programas de Alimentación Escolar (PAE), con el objeto de mejorar su asistencia a clases y contribuir a evitar la deserción escolar27. Si bien la mayoría de las escuelas incluyen legumbres, con una frecuencia de dos veces por semana en el almuerzo escolar, un estudio cualitativo realizado con los mensajes de las Guías Alimentarias 2013 observó una clara aceptación de su consumo en los niños y un rechazo a su preparación en el hogar por las madres de preescolares y escolares pequeños, indicando el apoco apoyo del hogar a la adquisición de este saludable hábito en los niños ${ }^{28}$.

\section{La educación alimentaria nutricional y el marketing social, componentes clave para el fomento de estilos de vida saludables en la población}

Aunque el año 1998, la Organización de las Naciones Unidas para la Alimentación y la Agricultura (FAO), ya identificaba a las escuelas como el objetivo principal para educar en nutrición, considerando que además de tener la responsabilidad de la educación en general, una nutrición apropiada es esencial para el desarrollo físico y mental de niños y adolescentes, en un estudio en 50 países identificó que las principales barreras que limitaban la implementación y la efectividad de la educación en nutrición en las escuelas eran la falta de formación en nutrición de los profesores (69\%); la inexistencia de materiales educativos apropiados para profesores y alumnos (61\%); la falta de apoyo político para incorporarla oficialmente en los planes y programas de estudios $(59 \%)$ y la falta de financiamiento $(43 \%)^{29}$.

Este Organismo Internacional patrocinó un importante estudio para incorporar la educación en nutrición en las 
escuelas entre los años 2001 al 2003, con la participación de 10 escuelas urbanas y rurales en 3 regiones del país. El estudio fue realizado por académicos del INTA con el apoyo del Ministerio de Educación. Aunque se desarrolló y validó materiales educativos para todos los niveles de enseñanza básica, este no fue incorporado en los planes y programas de estudios ${ }^{30}$.

En los documentos preparados para la Segunda Conferencia Mundial sobre Nutrición, la FAO vuelve a destacar el importante papel de la escuela en la formación de hábitos alimentarios saludables en los niños, a través de una educación en nutrición que cuente con el apoyo del ambiente escolar para lograr mejores resultados ${ }^{31}$. En Chile desde el año 2000 existen diversas experiencias de inserción de la educación alimentaria nutricional en las escuelas, logrando resultados alentadores en el aumento de consumo de alimentos saludables, como frutas, verduras, legumbres y lácteos ${ }^{32}$. El año 2014, una experiencia innovadora realizada por el Programa 5 al Día Chile y el INTA el año 2014, que incluye la entrega de frutas 3 veces por semana en escuelas públicas, ha logrado aumentar significativamente el consumo de legumbres 2 veces a la semana, con el apoyo de las acciones de educación en nutrición incluidas en la intervención ${ }^{33}$.

Un estudio realizado entre el año 2014 y 2015 en escuelas públicas de tres regiones del país, pudo demostrar que los niños expuestos a educación alimentaria nutricional inserta en los subsectores de aprendizaje y kiosco saludable mejoraron significativamente el consumo de legumbres $(28,3 \%)$, pescado $(39,6 \%)$, lácteos $(35,7 \%)$, frutas $(34 \%)$, verduras $(26,2 \%)$ y agua $(44 \%)^{34}$.

Hawkes y cols. ${ }^{35}$, plantearon el año 2015 que existían 4 aspectos clave para la prevención de la obesidad, que debían ser implementados en el marco de las políticas públicas: 1) proporcionar un ambiente que facilite el aprendizaje de preferencias saludables; 2 ) superar las barreras para facilitar la expresión de las preferencias saludables; 3) estimular a la población a reevaluar sus actuales preferencias poco saludables en el momento de la compra y 4) estimular la respuesta positiva de los sistemas de alimentación.

Sin embargo, a pesar de las recomendaciones de los Organismos Internacionales y de las positivas experiencias logradas por grupos académicos del país, en Chile no existe una política que permita incorporar la Educación Alimentaria Nutricional (EAN), como parte del currículum escolar. Si bien es cierto, el año 2012 se publicó la Ley sobre Composición Nutricional de los Alimentos y su Publicidad ${ }^{16}$, que establece la prohibición de vender alimentos con un alto contenido de calorías y nutrientes críticos (grasas saturadas, azúcar y sodio) en los establecimientos educacionales, sólo recomienda incluir actividades didácticas y físicas que contribuyan a desarrollar hábitos de una alimentación saludable en los niños, sin hacer referencia a los planes y programas de estudios del Ministerio de Educación, por lo que sigue siendo un tema pendiente de normar e incluir en la educación formal.

\section{CONCLUSIONES}

Los Organismos Internacionales han logrado demostrar la importancia de la escuela en el desarrollo de estilos de vida saludables, pero es poco probable que las tasas de obesidad infantil puedan disminuir sin políticas y programas establecidos oficialmente para apoyar la alimentación sana y la actividad física en todo el país.

\section{BIBLIOGRAFÍA}

1. Popkin BM, Gordon-Larsen P. The nutrition transition: worldwide obesity dynamics and their determinants. Int I Obes Relat Metab Disord. 2004; 28 (Suppl 3): S2-9.

2. Vio F, Albala C, Kain J. Nutrition transition in Chile revisited: mid-term evaluation of obesity goals for the period 20002010. Public Health Nutr 2008; 11(4): 405-412.

3. World Health Organization. Global Strategy on Diet, Physical Activity and Health. Geneva: WHO; 2004.

4. World Health Organization. Report of the Commission on Ending Childhood Obesity. WHO 2015.

5. Wang Y, Lim H. The global childhood obesity epidemic and the association between socio-economic status and childhood obesity. Int Rev Psychiatry 2012; 24(3): 176-188.

6. National Board of School Help and Scholarships. Plan Against Student Obesity. Santiago de Chile; 2016. Available in: http://contrapeso.junaeb.cl/wp-content/uploads/2017/03/ mapa_nutricional_2016.pdf

7. Crovetto M, Uauy $R$, Martins AP, Moubarac JC, Monteiro C. Consume food and drink products in Chile: impact on nutritional quality of the diet. Rev Méd Chil 2014; 142(7): 850-858.

8. Olivares S, Bustos Z, Lera L, Zelada ME. Nutritional status, food consumption and physical activity in schoolchildren of different socioeconomic levels in Santiago, Chile. Rev Med Chil 2007; 135(1): 71-78.

9. Olivares $S$, Yáñez R, Díaz N. Advertising of food and dietary behaviors in schoolchildren from 5th to 8th grade. Rev Chil Nutr 2003; 30 (1): 36-42.

10. The Republic of Chile. Ministry of Health. Law 20606 on Nutritional Composition of Food and its Advertising. Santiago: Official Gazette of Chile; July 6, 2012.

11. Bustos N, Kain J, Leyton B, Olivares S, Vio F. Collations habitually consumed by children of municipal schools: Motivations for their choice. Rev Chil Nutr 2010; 37(2): 178-83.

12. Bustos N, Kain J, Leyton B, Vio F. Changes in the pattern of food consumption in Chilean schoolchildren with the implementation of a healthy kiosk. Arch Latinoam Nutr 2011; 61(3): 302-307.

13. Crovetto M. Changes in food structure and apparent nutrient consumption of households in Greater Santiago 1988-1997. Rev Chil Nutr 2002; 29(1): 24-32.

14. Crovetto M, Uauy R, Martins AP, Moubarac JC, Monteiro C. Availability of ready-to-eat food products in Chilean households and their impact on diet quality (2006-2007). Rev Med Chil 2014; 142(7): 850-858.

15. Crovetto M, Uauy R. Evolution of expenditure on processed foods in the population of Greater Santiago in the last 20 years. Rev Med Chil 2012; 140(3): 305-312.

16. Olivares S, Zacarías I, González CG, Villalobos E. Process for formulating and validating food guides for the Chilean population. Rev Chil Nutr 2013; 40(3): 262-268.

17. Vio F, Salinas J, Lera L, González CG, Huenchupán MC. Knowledge and food consumption in school children, their 
parents and teachers: a comparative analysis. Rev Chil Nutr 2012; 39(3): 34-39.

18. Crockett SI, Sims LS. Environmental influences on children's eating. I Nutr Educ 1995; 27(5): 235-249.

19. Patrick $H$, Nicklas TA. A review of family and social determinants of children's eating patterns and diet quality. I Am Coll Nutr 2005; 24(2): 83-92.

20. Rodgers RF, Paxton SI, Massey R, Campbell KJ, Wertheim EH, Skouteris $H$, et al. Maternal feeding practices predict weight gain and obesogenic eating behaviors in young children: a prospective study. Int I Behav Nutr Phys Act 2013; 10(1):1-10.

21. Shloim N, Edelson LR, Martin N, Hetherington MM. Parenting styles, feeding styles, feeding practices, and weight status in 4-12 year-old children: A Systematic Review of the Literature. Front Psychol 2015;6.

22. Briefel $R$, Crepinsek $M$, Cabili $C$, Wilson A, Gleason P. School food environments and practices affect dietary behaviors of US public school children. I Am Diet Assoc 2009; 109(2): S91-107.

23. WHO/FAO. Diet, Nutrition and the prevention of chronic diseases. Geneva; WHO/FAO 2003.

24. Ministry of Health. Undersecretary of Public Health. Dietary Guidelines for the Chilean population. Castillo C, Uauy R, Atalah E, eds. Santiago; 1997.

25. Jury G, Urteaga C, Taibo M. Portions of exchange and chemical composition of food in the Chilean food pyramid. Santiago; 1997.

26. Chile's goverment. Ministry of Health; INTA / University of Chile; Life Chile. Guide to a healthy life. Santiago; 2005.

27. National Board of School Help and Scholarships. School Feeding Program. Santiago de Chile; 2016. Available at: https://www.junaeb.cl/programa-de-alimentacion-escolar

28. Olivares S, Zacarías I, González CG. Motivations and barriers of Chilean children: threats or opportunities for the implementation of the 2013 food guidelines? Nutr Hosp 2014; 30(2): 260-266.

29. Olivares S, Snel J, McGrann M, Glassauer P. Education in nutrition in primary schools. Food Nutr Agric 1998; 20: 5760.

30. Olivares S, Morón C, Zacarías I, Andrade M, Vio F. Nutrition education in basic schools in Chile. FAO 2003; Available from: http://www.fao.org/fileadmin/ user_upload/red-icean/ docs/Chile_icean en la escuela_educacion nutricion_2003_ REDICEAN.pdf.pdf

31 FAO. Promoting healthy diets through nutrition education and changes in the food environment: an international review of actions and their effectiveness. Background Paper for the International Conference on Nutrition. In 2013. p. 1-78.

32. Vio F, Zacarías I, Lera L, Benavides M, A G. Prevention of obesity in basic schools of Peñalolén: Food and nutrition component. Rev Chil Nutr 2011; 38: 268-276.

33. Evaluation of a fruit delivery program with nutritional education in rural public schools in the Metropolitan Region, Chile. Rev Chil Nutr 2014; 41(3): 228-235.

34. Bustos N. Effectiveness of an intervention in food and physical activity, aimed at controlling the increase of obesity in children belonging to public schools in three regions of the country. (doctoral thesis). University of Chile. In Santiago; 2017

35. Hawkes C, Smith TG, Jewell J, Wardle J, Hammond RA, Friel $S$, et al. Smart food policies for obesity prevention. Lancet 2015; 385(9985): 2410-2421. Available from: http://dx.doi. org/10.1016/S0140-6736(14)61745-1 